\title{
Magnetic Properties of Hexagonal RTIn Rare-Earth Intermetallics with Frustration
}

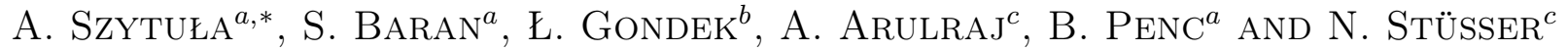 \\ ${ }^{a}$ M. Smoluchowski Institute of Physics, Jagiellonian University, Reymonta 4, 30-059 Kraków, Poland \\ ${ }^{b}$ Faculty of Physics and Applied Computer Science, AGH University of Science and Technology \\ al. Mickiewicza 30, 30-059 Kraków, Poland \\ ${ }^{c}$ BENSC Hahn-Meitner Institute, Glienicker Str. 100, D-14109 Berlin, Germany
}

\begin{abstract}
Magnetic properties, including magnetic structure, of some RTIn compounds $(\mathrm{R}=\mathrm{Tb}-\mathrm{Er} ; \mathrm{T}=\mathrm{Cu}, \mathrm{Ni}$, $\mathrm{Pd}, \mathrm{Pt}, \mathrm{Au})$ with the hexagonal ZrNiAl-type crystal structure are reported. Distribution of the rare earth atoms in the basal plane is similar to the kagomé-lattice. Magnetic moments are localized exclusively on the rare earth atoms and form different types of magnetic ordering. Experimental results are analyzed within the Ruderman-Kittel-Kasuya-Yosida and crystal electric field frames. Monte Carlo simulations, including the $J_{1}$ and $J_{2}$ exchange integrals between nearest and next nearest spins and Dzialoshinsky-Moriya interaction, were performed resulting in a number of magnetic phases, some of which are in good agreement with the magnetic ordering determined in the neutron diffraction experiments.
\end{abstract}

PACS numbers: 71.20.Lp, 75.30.Kz

\section{Introduction}

Equiatomic intermetallic compounds RTX $(\mathrm{R}=$ rare earths, $\mathrm{T}=$ transition metals; $\mathrm{X}=$ elements of the $4 \mathrm{th}$ or 5th main group) have attracted considerable interest in the last two decades due to their greatly varying physical properties [1-4]. Nowadays more than 1000 compounds are known [4]. They crystallize in more than 40 different structure types [5], however majority of the RTX compounds crystallizes in the following three ones: orthorhombic TiNiSi- and hexagonal LiGaGe- or ZrNiAl-type of crystal structure [6].

The large variety of magnetic behaviors found in these compounds arises from the competition of long-range interactions between the $4 f$ ions in the presence of magnetocrystalline anisotropy. This competition results in frustrated magnetic states such as collinear incommensurate, long-period commensurate or antiphase magnetic structures. The anisotropy plays a crucial role in the existence and temperature dependence of these structures according to the uniaxial (Ising-like) or planar $(X Y)$ character of the compounds. The magnetic structures of the RTIn compounds with the ZrNiAl-type structure are good illustration for the various effects of frustration.

In the $\mathrm{ZrNiAl}$-type of crystal structure (space group $P \overline{6} 2 m$ ) the crystal lattice could be thought as built up of two types of planes, with and without rare-earth atoms,

* corresponding author; e-mail: szytula@if.uj.edu.pl alternating along the $c$-axis. The distribution of the atoms in the planes is similar to the kagomé lattice (Fig. 1). In case of an antiferromagnetic coupling between the nearest neighbor rare earth magnetic moments frustration of the magnetic interactions is induced by this topology.

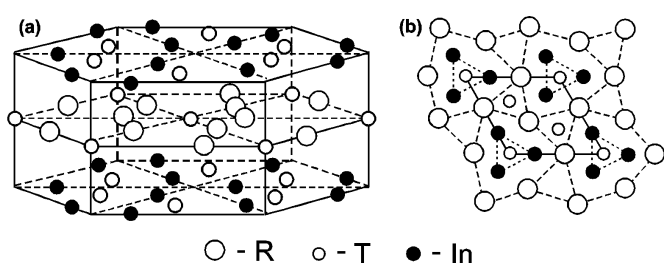

Fig. 1. (a) Hexagonal ZrNiAl-type crystal structure and $(b)$ its projection into the basal plane.

In this work the results obtained for the RTIn $(\mathrm{T}=\mathrm{Ni}$, $\mathrm{Cu}, \mathrm{Pd}, \mathrm{Pt}$ and $\mathrm{Au}$ ) compounds with heavy rare earth elements $(\mathrm{R}=\mathrm{Tb}-\mathrm{Er})$ are presented.

\section{Results and discussion}

Neutron diffraction patterns were recorded using the E6 diffractometer at BENSC (Hahn-Meitner Institute, Berlin) with the incident neutron wavelength $2.44 \AA$ at several temperatures between 1.5 and $60 \mathrm{~K}$. The data concerning structural and magnetic properties of the studied compounds are summarized in Table I. 


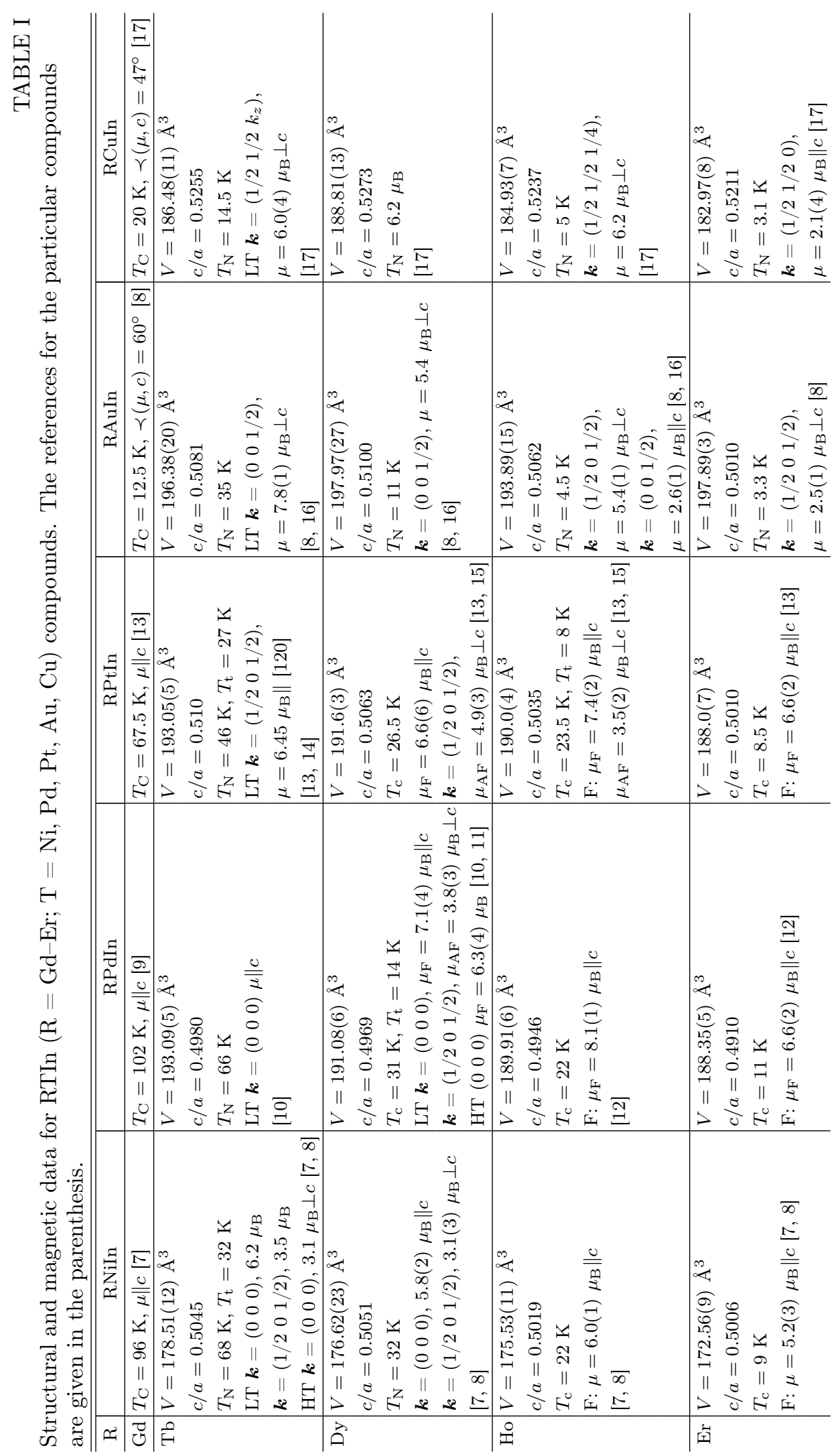


In the studied rare-earth intermetallics the interatomic distances along the $a$ - as well as the $c$-axis are large, therefore two mechanisms in which the $4 f$ moments can interact in an indirect way have been taken into account. In the Ruderman-Kittel-Kasuya-Yosida (RKKY) interaction the magnetic coupling proceeds by means of spin polarization of conduction electrons and the interaction is proportional to the de Gennes factor $\left(g_{J}-1\right)^{2} J(J+1)$. The critical temperatures of the magnetic ordering scale well with the de Gennes factor for the RTIn compounds with $\mathrm{T}=\mathrm{Cu}, \mathrm{Pt}, \mathrm{Pd}$ and $\mathrm{Ni}$ but for the RTIn compounds with $\mathrm{T}=\mathrm{Ni}, \mathrm{Pd}$ and $\mathrm{Pt}$ the values of the critical temperatures are considerable higher than for those with $\mathrm{T}=\mathrm{Cu}$ and $\mathrm{Au}$ (Fig. 2).

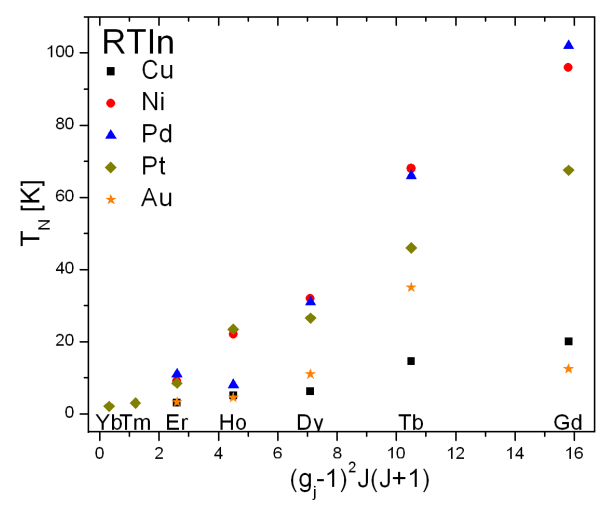

Fig. 2. Magnetic ordering temperatures $T_{\mathrm{m}}$ for RTIn $(\mathrm{R}=\mathrm{Gd}-\mathrm{Yb} ; \mathrm{T}=\mathrm{Ni}, \mathrm{Cu}, \mathrm{Pd}$ and $\mathrm{Au})$ versus the de Gennes scaling factor $d G=\left(g_{J}-1\right)^{2} J(J+1)$.

In order to get a deeper insight into magnetic ordering in these compounds some basic studies using Monte Carlo simulations were performed. The phase diagram calculations were made considering the following Hamiltonian:

$$
\begin{aligned}
H & =-J_{1} \sum_{i, i+1} \boldsymbol{S}_{i} \cdot \boldsymbol{S}_{i+1}-J_{2} \sum_{i, i+2} \boldsymbol{S}_{i} \cdot \boldsymbol{S}_{i+2} \\
& -\boldsymbol{D} \sum_{i, i+1} \boldsymbol{S}_{i} \times \boldsymbol{S}_{i+1}
\end{aligned}
$$

where $J_{1}$ and $J_{2}$ are the exchange constants between the nearest and next nearest spins, respectively, and $\boldsymbol{D}$ is a constant vector defining Dzyaloshinskii-Moriya interaction (DM). Classical spins lying within the basal plane and the $\boldsymbol{D}$ vector perpendicular to the basal plane were assumed. The calculations were carried out for a honeycomb-like lattice containing 90 spins [18]. Including nearest and next nearest neighbors interactions $\left(J_{1}, J_{2}\right)$, five magnetic phases can be established, however, only three of them are long-range order ones: ferromagnetic $(\mathrm{F}), 120^{\circ}$-type antiferromagnetic $\left(\mathrm{AF}_{1}\right)$ and modulated antiferromagnetic $\left(\mathrm{AF}_{2}\right)$ phase. The $\mathrm{F}$ phase exists when $J_{1}>0$ and $J_{2}<0.7 J_{1}\left(J_{1}>0\right.$ establishes ferromagnetic phase). This type of magnetic structure is observed in TbPdIn [10]. The $\mathrm{AF}_{1}$ phase exists when both $J_{1}$ and $J_{2}$ are negative. The magnetic moments of the nearest and next nearest neighbors form the angle of $120^{\circ}$. Such ordering was found for TbNiIn, DyNiIn, TbAuIn, DyAuIn, and ErAuIn [8]. Taking into account an additional exchange integral along the $c$-axis, a new modulated magnetic structure described by the propagation vector $\boldsymbol{k}=(0,0,1 / 2)$ is obtained for $J_{\mathrm{c}}<0$. In the $\mathrm{AF}_{2}$ phase the magnetic ordering is described by the propagation vector $\boldsymbol{k}=(1 / 3,1 / 3)$. The magnetic moments of the nearest neighbors form the angle of $120^{\circ}$, while the second neighbors order ferromagnetically. This order was observed for: RAgSi ( $\mathrm{R}=\mathrm{Dy}, \mathrm{Ho})$ and RAgGe $(\mathrm{R}=\mathrm{Tb}, \mathrm{Dy}, \mathrm{Ho})[19,20]$. There are also two phases with no long-range magnetic order possible for $J_{1}>0$ : for $-8 J_{1}<J_{2}<-0.7 J_{1}$ the one connected with destruction of the ferromagnetic order of the nearest spins and for $J_{2}<-8 J_{1}$ in which the second neighbors order antiferromagnetically with the angle of $120^{\circ}$ between the magnetic moments. Including the DM interaction and considering it as a small perturbation to the superexchange $(D / J \ll 1)$ the following magnetic structures with long-range order were obtained for different $D / J_{1}$ values: $\boldsymbol{k}=(0.02,0.02)$ for $\left|D / J_{1}\right|=0.1, \boldsymbol{k}=(1 / 6,1 / 6)$ for $\left|D / J_{1}\right|=1$ and $\boldsymbol{k}=(1 / 3,1 / 3)$ for $\left|D / J_{1}\right|=\infty$.

The other factor influencing the magnetic ordering in the studied compounds is the crystal electric field $(\mathrm{CF})$. In the compounds with the ZrNiAl-type crystal structure the $\mathrm{CF}$ acting on the rare earth ions has an orthorhombic symmetry and can be described by the Hamiltonian [21]:

$$
\begin{gathered}
\mathrm{H}_{\mathrm{CF}}=\mathrm{B}_{2}^{0} \mathrm{O}_{2}^{0}+\mathrm{B}_{2}^{2} \mathrm{O}_{2}^{2}+\mathrm{B}_{4}^{0} \mathrm{O}_{4}^{0}+\mathrm{B}_{4}^{2} \mathrm{O}_{4}^{2}+\mathrm{B}_{4}^{4} \mathrm{O}_{4}^{4} \\
+\mathrm{B}_{6}^{0} \mathrm{O}_{6}^{0}+\mathrm{B}_{6}^{2} \mathrm{O}_{6}^{2}+\mathrm{B}_{6}^{4} \mathrm{O}_{6}^{4}+\mathrm{B}_{6}^{6} \mathrm{O}_{6}^{6},
\end{gathered}
$$

where $\mathrm{B}_{n}^{m}=\mathrm{A}_{n}^{m}\left\langle r^{n}\right\rangle \theta_{n}, A_{n}^{m}$ (or $\mathrm{B}_{n}^{m}$ ) are so-called crystal field parameters, $\left\langle r^{n}\right\rangle$ mean the average over the radial part of the $4 f$ wave function, $\theta_{n}$ are the Stevens multiplication factors (usually the notation $\theta_{2}=\alpha, \theta_{4}=\beta$, $\theta_{6}=\gamma$ is used). In many cases the $n=2$ term is dominant and the direction of the magnetic moments in the crystal is determined by the sign of the $\alpha$-factor of the corresponding $R^{3+}$ ion, which is negative for $\mathrm{Tb}^{3+}$, $\mathrm{Dy}^{3+}, \mathrm{Ho}^{3+}$ ions but positive for $\mathrm{Er}^{3+}$. The above suggests that with increasing number of $4 f$ electrons a change in the direction of the magnetic moment should be observed between Ho- and Er-compounds. This is fulfilled only for the RCuIn series: the magnetic moments lie in the basal plane for $\mathrm{R}=\mathrm{Tb}$ and Ho while they are parallel to the $c$-axis for $\mathrm{R}=\mathrm{Er}$. In RNiIn series this change is observed between Dy- and Ho-compounds. It is worth to notice that both factors, $\alpha$ and $\beta$, for $\mathrm{Dy}^{3+}$ and $\mathrm{Ho}^{3+}$ are negative. Therefore, the difference in the anisotropy between DyNiIn and HoNiIn points to an important role of the term with the $\gamma$-factor which is positive for $\mathrm{Dy}^{3+}$ and negative for $\mathrm{Ho}^{3+}$. For other compounds a strong influence of the $\mathrm{T}$ element on the direction of the magnetic moment is observed: for $\mathrm{T}=\mathrm{Pd}$ and $\mathrm{Pt}$ the magnetic moments are parallel to the $c$-axis, while for $\mathrm{T}=\mathrm{Au}$ they lie in the basal plane. For RPtIn the magnetic data for 
a single crystal are reported by Morosan et al. [13]. This data and our neutron diffraction results (summarized in Table II) indicate a change in the magnetic properties, including the direction of the magnetic moment.

\section{TABLE II}

Magnetic data for the RPtIn single crystals from Ref. [13], the calculated values of the crystal field parameters $B_{2}^{0}$ and $B_{2}^{2}$, and the direction of the magnetic moment from the neutron diffraction data.

\begin{tabular}{c|c|c|c|c}
\hline \hline & $\mathrm{Tb}$ & $\mathrm{Dy}$ & Ho & Er \\
\hline$T_{\mathrm{m}}[\mathrm{K}]$ & 46 & 26.5 & 23.5 & 8.5 \\
$\mathrm{TMM}$ & $\mathrm{AF}$ & $\mathrm{F}$ & $\mathrm{F}$ & $\mathrm{F}$ \\
$\theta_{a}[\mathrm{~K}]$ & -38.1 & -2.2 & 7.5 & 14.5 \\
$\theta_{c}[\mathrm{~K}]$ & -29.2 & -29.0 & -27.8 & -9.6 \\
$B_{2}^{0}[\mathrm{~K}]$ & -2.04 & -0.62 & -0.36 & +0.10 \\
$B_{2}^{2}[\mathrm{~K}]$ & +0.27 & +0.53 & +0.62 & +0.48 \\
$\mathrm{DMM}$ & $\perp c$ & $\theta=37^{\circ}$ & $\theta=25^{\circ}$ & $\| c$
\end{tabular}

$T_{\mathrm{m}}$ - magnetic ordering temperature, TMM - type of magnetic ordering, $\theta_{a b}, \theta_{c}-$ anisotropic paramagnetic CurieWeiss temperatures in $a b$ plane and $c$-axis, DMM - direction of the magnetic moment, $B_{2}^{0}, B_{2}^{2}-$ crystal field parameters, $\theta$ - angle between the direction of the magnetic moment and the $c$-axis

The equations below allow us to determine the values of the CF parameters $B_{2}^{0}$ and $B_{2}^{2}$ :

$$
\begin{aligned}
& \theta_{a}=\theta_{\text {ave }}+\frac{(2 J-1)(2 J+3)}{10}\left(B_{2}^{0}+B_{2}^{2}\right), \\
& \theta_{c}=\theta_{\text {ave }}+\frac{(2 J-1)(2 J+3)}{10}\left(B_{2}^{0}-B_{2}^{2}\right) .
\end{aligned}
$$

The obtained results are listed in Table II. The change in the $B_{2}^{0}$ and $B_{2}^{2}$ values with increasing number of $4 f$ electrons are responsible for the changes in the direction of the magnetic moment. Different values of the CF parameters were obtained for RNiIn and RAuIn series from the calculations basing on the point charge model [22]. This results show the change in the $B_{2}^{0}$ and $B_{2}^{2}$ values from positive for $\mathrm{R}=\mathrm{Tb}$, Dy and Ho to negative for $\mathrm{R}=$ Er. The agreement between the direction of the rare-earth magnetic moment and $B_{2}^{0}$ signs is observed for some compounds.

\section{Summary}

1. The ordering temperatures $T_{\text {ord }}$ of the RTIn $(\mathrm{R}=$ $\mathrm{Gd}-\mathrm{Er}, \mathrm{T}=\mathrm{Ni}, \mathrm{Pd}, \mathrm{Pt}, \mathrm{Cu}$ ) compounds scale fairly well with the de Gennes factor. This suggests that the RKKY interaction stabilizes the long-range magnetic order in these compounds. The deviation observed for RAuIn series suggests a strong influence of the crystal electric field.
2. The magnetic structures observed in RTIn are in good agreement with the ones calculated in the $X Y$ model including the Dzyaloshinskii-Moriya interactions and geometrical frustration. However, this model does not explain the magnetic structure described by the propagation vector $\boldsymbol{k}=(1 / 2,0,1 / 2)$.

3. Influence of the crystal electric field on the magnetic order is observed. It results in different orientation of the rare-earth magnetic moment in respect of the $c$-axis and lowering their values in comparison to the free ion ones.

4. With increasing temperature the magnetic structure of DyPdIn, DyPtIn and HoPtIn changes from a canted one to a collinear ferromagnetic one with the magnetic moments parallel to the $c$-axis which indicates the influence of higher order $\mathrm{CF}$ terms.

5. For the full interpretation of the observed magnetic structures it is necessary to collect precise data concerning $\mathrm{CF}$ parameters.

\section{References}

[1] A. Szytuła, J. Leciejewicz, Handbook of Crystal Structures and Magnetic Properties of Rare Earth Intermetallics, CRC Press, Boca Raton (FL) 1994.

[2] A. Szytuła, in: Handbook of Magnetic Materials, Ed. K.H.J. Buschow, Vol. 6, North-Holland, Amsterdam 1991, p. 85.

[3] D. Gignoux, D. Schmitt, in: Handbook of Magnetic Materials, Vol. 10, Noth-Holland, Amsterdam 1997, Ch. 2, p. 239.

[4] A. Szytuła, Crystal Structures and Magnetic Properties of RTX Rare Earth Intermetallics, Wydawnictwo Uniwersytetu Jagiellońskiego, Kraków 1998.

[5] E. Parthé, B. Chabot, in: Handbook on the Physics and Chemistry of Rare Earths, Eds. K.A. Gschneidner Jr., L. Eyring, Vol. 7, North-Holland, Amsterdam 1984, p. 113.

[6] M.L. Fornasini, F. Merlo, J. Alloys Comp. 219, 63 (1995).

[7] Yu.B. Tyvanchuk, Ya. Kalychak, Ł. Gondek, M. Rams, A. Szytuła, Z. Tomkowicz, J. Magn. Magn. Mater. 277, 368 (2004).

[8] Ł. Gondek, A. Szytuła, S. Baran, J. Hernandez-Velasco, J. Magn. Magn. Mater. 272-276, e443 (2004).

[9] M. Bałanda, A. Szytuła, M. Guillot, J. Magn. Magn. Mater. 247, 345 (2002).

[10] P. Javorsky, P. Svoboda, S. Nishigori, M. Hofmann, N. Stüsser, Acta Phys. Slov. 48, 767 (1998).

[11] P. Javorsky, S. Nishigori, B. Ouladdiaf, Physica B, 276-278, 730 (2000).

[12] Ł. Gondek, S. Baran, A. Szytuła, D. Kaczorowski, J. Hernandez-Velasco, J. Magn. Magn. Mater. 285, 272 (2005).

[13] E. Morosan, S.L. Bud'ko, P.C. Canfield, M.S. Torikachvili, A.H. Lacerola, Phys. Rev. B 72, 014425 (2005). 
[14] O. Garlea, E. Morosan, S.L. Bud'ko, J.L. Zarestky, P.C. Canfield, C. Stassis, J. Appl. Phys. 95, 6921 (2004).

[15] S. Baran, Ł. Gondek, J. Hernandez-Velasco, D. Kaczorowski, A. Szytuła, J. Magn. Magn. Mater. 305, 196 (2006); 300484 (2006).

[16] A. Szytuła, W. Bażela, Ł. Gondek, T. Jaworska-Gołąb, B. Penc, N. Stüsser, A. Zygmunt, J. Alloys Comp. 336, 1 (2002).

[17] A. Szytuła, A. Arulraj, S. Baran, T. Jaworska-Gołąb, B. Penc, N. Stüsser, Yu. Tyvanchuk, A. Zarzycki, Acta Phys. Pol. A 113, 1185 (2008).

[18] Ł. Gondek, A. Szytuła, J. Alloys Comp. 442, 108 (2007).
[19] S. Baran, M Hofmann, J. Leciejewicz, B. Penc, M. Ślaski, A. Szytuła, J. Alloys Comp. 281, 92 (1998).

[20] S. Baran. M Hofmann, J. Leciejewicz, B. Penc, M. Ślaski, A. Szytuła, A. Zygmunt, J. Magn. Magn. Mater. 222, 277 (2000).

[21] M.T. Hutchings, Solid State Phys. 16, 227 (1964).

[22] Ł. Gondek, Ph.D. Thesis, Jagiellonian University, Kraków 2004. 